\title{
Cytokeratin $8 / 18$ expression indicates a poor prognosis in squamous cell carcinomas of the oral cavity
}

\author{
Thomas Fillies*1, Richard Werkmeister ${ }^{2}$, Jens Packeisen ${ }^{3}$, Burkhard Brandt ${ }^{4}$, \\ Philippe Morin ${ }^{5}$, Dieter Weingart ${ }^{5}$, Ulrich Joos ${ }^{1}$ and Horst Buerger ${ }^{6}$
}

\begin{abstract}
Address: ${ }^{1}$ Department of Cranio-Maxillofacial Surgery, University of Muenster, Waldeyerstrasse 30, 48129 Muenster, Germany, ${ }^{2}$ Department of Oral and Maxillofacial Surgery, Central German Armed Forces Hospital, Rübenacher Str. 170, 56072 Koblenz, Germany, ${ }^{3}$ Institute of Pathology, Klinikum Osnabrueck, Germany, ${ }^{4}$ Institute of Tumor Biology, Center of Experimental Medicine, University Medical Center Hamburg-Eppendorf, Martinistr. 52, 20146 Hamburg, Germany, ${ }^{5}$ Department of Maxillofacial Surgery, Katharinenhospital Stuttgart, Kriegsbergstrasse 60, 70174 Stuttgart, Germany and 'Institute of Pathology, University of Muenster, Domagkstraße 17, 48149 Muenster, Germany

Email: Thomas Fillies* - fillies@uni-muenster.de; Richard Werkmeister - Rwerkmeis@aol.com; Jens Packeisen - jpackeisen@pathoweb.de; Burkhard Brandt - bu.brandt@uke.uni-hamburg.de; Philippe Morin - p.morin@katharinenhospital.de;

Dieter Weingart - weingart.mkg@katharinenhospital.de; Ulrich Joos - joos@uni-muenster.de; Horst Buerger - burgerh@uni-muenster.de

* Corresponding author
\end{abstract}

Published: 13 January 2006

BMC Cancer 2006, 6:10 doi:10.1186/147I-2407-6-10
Received: II May 2005

Accepted: 13 January 2006

This article is available from: http://www.biomedcentral.com/I47/-2407/6/10

(C) 2006 Fillies et al; licensee BioMed Central Ltd.

This is an Open Access article distributed under the terms of the Creative Commons Attribution License (http://creativecommons.org/licenses/by/2.0), which permits unrestricted use, distribution, and reproduction in any medium, provided the original work is properly cited.

\begin{abstract}
Background: Intermediary filaments are involved in cell motility and cancer progression. In a variety of organs, the expression of distinct intermediary filaments are associated with patient prognosis. In this study, we seeked to define the prognostic potential of cytokeratin and vimentin expression patterns in squamous cell carcinomas (SCC's) of the oral cavity.

Methods: 308 patients with histologically proven and surgically treated squamous cell carcinomas of the oral cavity were investigated for the immunohistochemical expression of a variety of intermediary filaments including high- and low-molecular weight cytokeratins (Ck's), such as $\mathrm{Ck} 5 /$ 6, Ck 8/18, Ck I, CK 10, Ck 14, Ck 19 and vimentin, using the tissue microarray technique. Correlations between clinical features and the expression of Cytokeratins and vimentin were evaluated statistically by Kaplan-Meier curves and multivariate Cox regression analysis.

Results: The expression of $\mathrm{Ck} 8 / \mathrm{I} 8$ and $\mathrm{Ck} 19$ were overall significantly correlated with a poor clinical prognosis $(C k 8 / 18 p=0.04 ; C k 19 p<0.01)$. These findings could also be reproduced for $\mathrm{Ck} 8 / 18$ in primary nodal-negative SCC's and held true in multivariate-analysis. No significant correlation with patient prognosis could be found for the expression of the other cytokeratins and for vimentin.

Conclusion: The expression of $\mathrm{Ck} 8 / 18$ in SCC's of the oral cavity is an independent prognostic marker and indicates a decreased overall and progression free survival. These results provide an extended knowledge about the role of intermediary filament expression patterns in SCC's.
\end{abstract}

\section{Background}

Intermediary filaments, like cytokeratins are essential intracellular components, underlying or reflecting distinct cellular properties and differentiation stages in epithelial organs. The proteins of the cytokeratin family are epithelium specific expressed [1] as low and high-molecular 
weight, resp. acid and basic polypeptides. Squamous, stratified epithelium is usually characterized by the expression of CK 5 , which is found mainly in the basal cell layers and $\mathrm{Ck} 5$ is associated with the proliferative potential of these cells $[2,3]$. The intermediary cell layers show an additional expression of Ck's 1 and 10, which are regarded as signs of cellular differentiation [2]. In contrast, glandular epithelia reveal the expression of lowmolecular weight cytokeratins Ck's 8/18 and 19 as typical features - expression pattern of Ck $8 / 18$ is rather uncommon in mature squamous epithelium. Ck 19 expressed heterogeneously in the basal cell layers of stratified squamous epithelium [2,3]. Suprabasal expression of Ck 19 seems to be correlated with premalignant transformation in oral epithelium [4]. The expression of vimentin is mainly regarded as sign of a mesenchymal differentiation. However, vimentin-positively has repeatedly reported in various carcinomas and was interpreted as sign of an epithelial-mesenchymal transition, indicating an increased metastatic potential [5-9].

Recently, it could be shown that the induction of Ck 8/18 expression in non-malignant buccal mucosa cells resulted in a significant change of phenotypic characteristics after Ck 8/18 transfection [10]. These changes included an increased cellular motility, which might give first hints for an increased tumour aggressiveness and poor patient prognosis. However, these findings have not been transferred on a clinical level, yet. Therefore, the significance of these findings in a clinical context, especially in regard to the prognostic importance is unclear.

It was therefore the aim of this study to evaluate the expression of intermediary filaments in SCC's in relation to clinico-pathological features, especially for prognostic purposes by the use of tissue micro arrays and immunohistochemistry.

\section{Methods}

Patients with histologically proven squamous cell carcinoma of the oral floor treated surgically were eligible for the study. All patients underwent surgical treatment including radical resection of the whole tumour with a free histopathological margin of at least $4 \mathrm{~mm}$ from the tumour borders. Selective neck dissection of Level I, II, III and $\mathrm{V}$ was performed in case of suspect results in preoperative tumourstaging by computertomography or sonography examination or in case of tumour size over $2 \mathrm{~cm}$, bilateral selective neck dissection was performed when the tumour spread the midline (according to the recommendation of Robins et al. 2002 [11]). Radiotherapy was performed when lymph node metastases were detected histologically. All tumours were classified postsurgically according to the TNM system $6^{\text {th }}$ eds. UICC 2002 [12].

\section{Method}

Tumour specimens of 308 patients were investigated for the expression of Ck 5/6, Ck 8/18, Ck 1, CK 10, Ck 14, Ck 19 and vimentin (Table 1) by means of the tissue micro array (TMA) technique.

For tissue micro array (TMA) construction, two punch biopsies with a diameter of $0.6 \mathrm{~mm}$ from each donor block were taken and transferred into the new acceptor block. TMA construction was performed by using a special tissue micro array instrument (Beecher Instruments, New Jersey, USA), according to standard protocols $[13,14]$.

Immunohistochemistry was performed on $4-\mu$ m-thick TMA sections. After deparaffinization and rehydration, endogenous peroxidase activity was blocked for $30 \mathrm{~min}$ utes in methanol containing $0.3 \%$ hydrogen peroxide. After antigen retrieval, a cooling-off period of 20 minutes preceded the incubation of the primary antibody. Thereafter, the catalyzed signal amplification system (DAKO, Glostrup, Denmark) was used for Ck 5/6, Ck 8/18, Ck 1, CK 10, Ck 14, Ck 19 and vimentin staining according to the manufacturer's instructions.

All antibodies were detected by a standard avidin-biotin complex method with a biotinylated rabbit anti-mouse antibody (DAKO) and an avidin-biotin complex (DAKO). The stainings were developed with diaminobenzidine or alternatively using LSAB/AP (DAKO). Before the slides were mounted, all sections were counterstained for $45 \mathrm{sec}$ onds with hematoxylin and dehydrated in alcohol and xylene. Appropriate negative controls (obtained by omis-

Table I: Antibodies, company, clone, pre-treatment and dilution of used antibodies.

\begin{tabular}{lllll}
\hline Antibody & Company & Clone & Pre-treatment & Dilution \\
\hline Ck $5 / 6$ & DAKO, Glostrup, Denmark & D5/I6B4 & Autoclave I0 min & $1: 80$ \\
Ck 8/18 & Dianova, Hamburg, Germany & K8.8/DC10 & Autoclave 10 min, citrate buffer & $1: 40$ \\
Ck I & Novocastra, Newcastle, UK & $34 \beta B 4$ & Steamer 30 min, citrate buffer & $1: 150$ \\
Ck 10 & DAKO, Glostrup, Denmark & DE-K10 & Steamer 30 min, citrate buffer & $1: 400$ \\
Ck 14 & Dianova Hamburg, Germany & LL002 & Steamer 30 min, citrate buffer & $1: 50$ \\
Ck 19 & Quartett GmbH, Berlin, Germany & KS19.1 & Steamer 30 min, citrate buffer & $1: 80$ \\
Vimentin & Ventana Inc., Tucson, USA & V9 & Steamer 30 min, citrate buffer & $1: 1000$ \\
\hline
\end{tabular}


Table 2: Tumour patient cases in this study

\begin{tabular}{ll}
\hline Age (mean) & 58 years (range $31-90$ years) \\
\hline Sex & 240 men, 68 women \\
Follow-up (mean) & 64 months (range $4-$ I8I months) \\
T stadium & I 35 TI tumours \\
& I 25 T2 tumours \\
& I5 T3 tumours \\
& 33 T2 tumours \\
& 212 No lymph node negative \\
& 57 NI lymph node positive carcinoma \\
N stadium & 39 N2 lymph node positive carcinoma \\
& 54 well differentiated tumours (GI) \\
& 214 moderately differentiated tumours (G2) \\
Grading & 40 weakly differentiated tumours (G3)
\end{tabular}

sion of the primary antibody) and positive controls were used throughout.

The expression of Ck 5/6, Ck 8/18, Ck 1, CK 10, Ck 14, Ck 19 and vimentin were determined independently by two pathologists (HB and JP). Both pathologists determined the percentage of positive cells in each core.

The percentage was classified for Cytokeratin 19 in 3 groups (0\% no Expression, 1:1-50\% moderate expression, $2>50 \%$ high expression) and Ck 5/6, Ck 8/18, Ck 1, CK 10, Ck 14 and vimentin in two groups (0: no Expression, $1: \geq 1 \%$ positive expression). The mean percentage value of the two cores representing one tumour and were used for further evaluation.

\section{Statistical analysis}

The TNM-stage, the histological differentiation and the expression of Ck 5/6, Ck 8/18, Ck 1, CK 10, Ck 14, Ck 19 and vimentin were related to the duration of the progression-free and the overall survival. The measurement of time started from the date of surgery to the date of histologically proven recurrent or metastatic carcinoma or disease related death, respectively. Patients who died from intercurrent disease during the trial were censored at the date of death. Patients lost to follow-up were censored at the date of the last examination.

Correlations between clinical features and the expression of cytokeratins were evaluated statistically by chi-square tests. The overall survival curves were constructed according to Kaplan and Meier [15]. The Log-Rank test was used to assess differences between groups and the multivariate survival analysis was performed with Cox regression [16], a p-value $<0.05$ was considered to be significant.

\section{Results}

The series comprised 308 patients (240 men and 68 women) with a median age of 58 years (range 31-90). All
Table 3: Expression profile of cytokeratins

\begin{tabular}{lll}
\hline Antibody & No Expression & postive Expression \\
\hline CK $5 / 6$ & 123 & 169 \\
CK8/18 & 133 & 154 \\
CK I & 147 & 121 \\
CK 10 & 177 & 91 \\
CK I4 & 159 & 107 \\
Ck 19 & 149 & 107 \\
Vimentin & 237 & 24 \\
\hline
\end{tabular}

tumours were classified post surgically according to the TNM system [12]. Patients were clinically evaluated in our routine follow-up for 3-10 years. Clinical and tumour details of the patients with oral squamous cell cancer are shown in Table 2.

On average $90 \%$ of all cores could be evaluated for the expression of intermediate filaments.

Immunohistochemical examination showed that $\mathrm{Ck} 8 /$ 18, Ck 5/6, Ck 1, CK 10, Ck 14, Ck 19 and vimentin reactivity was confined homogeneously to the cytoplasma of neoplastic cells. The expression levels did not differ significantly for the vast majority of the cases between the two tumour cores representing one case, indicating a rather homogeneous expression. No correlation was found between Ck 1, Ck 5/6, Ck 10, Ck 14, vimentin and the survival prognosis.

Detectable levels of $\mathrm{Ck} 8 / 18$ (Ck 8/18 $\geq 1 \%$ ) were found in $54 \%(154 / 287)$ of the oral SCCs. Detectable levels of Ck 19 (Ck $19 \geq 1 \%$ ) were found in 37,5\% (96/256) of the cases, high levels of Ck 19 expression ( $\mathrm{Ck} 19>50 \%$ ) were found in $4,2 \%(11 / 256)$ of the investigated tumour specimens (Table 3 ). The immunohistochemical staining is shown in Figure 1.

Ck 5/6 and Ck 14 were significantly correlated with the tumour size and lymph node status in the Chi-square Test (Table 4 ) and between each other $(\mathrm{p}<0.01)$. No correlation was found between tumour size, tumour differentiation, lymph node status and the expression of Ck 8/18, Ck 1, Ck 10, Ck 19 and vimentin in Chi-square test.

Log-rank test of Ck 8/18 and Ck 19 expression showed a significantly decreased overall survival of carcinomas with a Ck 8/18 expression (Ck 8/18 $\geq 1 \%(\mathrm{n}=154), \mathrm{p}<0.04$,

Table 4: Chi-square Test of Ck 5-6 and Ck 14

\begin{tabular}{lcc}
\hline Prognosticator & Node status $P$ value & Tumour size $P$ value \\
\hline Ck 5-6 & 0.045 & 0.026 \\
Ck 14 & 0.021 & 0.037
\end{tabular}




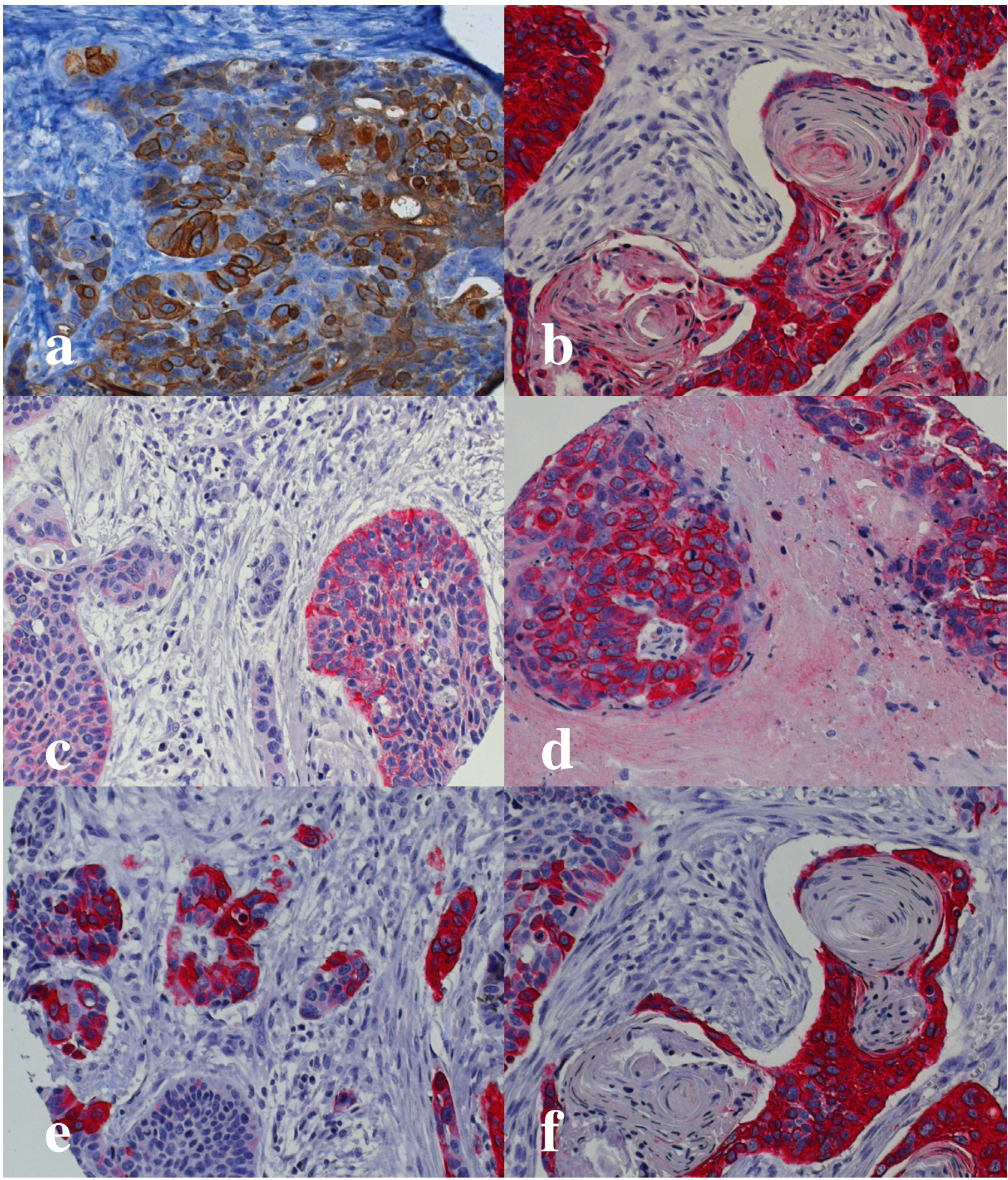

Figure I

Immunohistochemical staining of oral squamous cell carcinoma with cytokeratin cytokeratin antibodies. a) cytokeratin 5/6 Antibody (magnification 20x) b) cytokeratin 19 antibody (magnification 20x) c) cytokeratin 8/18 antibody (Magnification 10x) d) cytokeratin 8/18 antibody (magnification 20x) e) cytokeratin 14 antibody (magnification 20x) d) cytokeratin I4 antibody (magnification 20x). 


\section{Cytokeratin 8/18 expression}

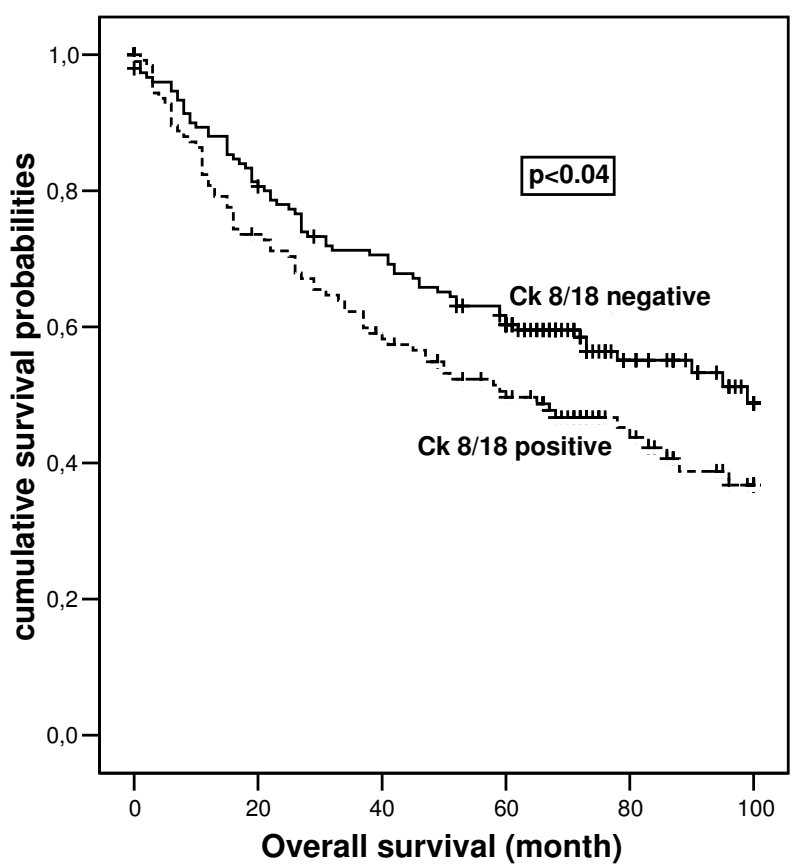

Figure 2

Overall survival of patient with oral squamous cell carcinoma in dependence on Cytokeratin 8-18 expression calculated by the Kaplan-Meier method.

Fig. 2) and a significant decreased overall survival associated with increased expression level of CK 19 (CK $19<1 \%$ $(\mathrm{n}=149), \mathrm{Ck} 19=1-49 \%(\mathrm{n}=96), \mathrm{Ck} 19>50 \%(\mathrm{n}=11)$, $\mathrm{p}<0.01$, Fig. 3)

In the subgroup of node negative oral squamous cell cancer the expression of $\mathrm{Ck} \mathrm{8/18}$ and the high expression of Ck 19 were also correlated with a poor overall survival (Ck 8/18, p < 0,04, Fig. 4) (Ck 19, p < 0.01). No influence of vimentin coexpression on the overall survival prognosis of Ck 8/18 and Ck 19 positive carcinomas was observed.

All carcinomas with an expression of Ck 8/18 and Ck 19 were reviewed and re-evaluated on whole sections. This was done in order to exclude the possibility of a misinterpretation and misclassification of dedifferentiated salivary gland carcinomas as poorly differentiated SCC's. However, in all these tumours the diagnosis of a primary salivary gland carcinoma could be ruled out.

In multivariate Cox analysis we compared the overall survival according to clinically established prognostic factors tumour size and nodal status with the expression of $\mathrm{Ck} 8$ / 18, Ck 5/6, Ck 1, CK 10, Ck 14 and Ck 19 (Table 5). Three

\section{Cytokeratin 19 expression}

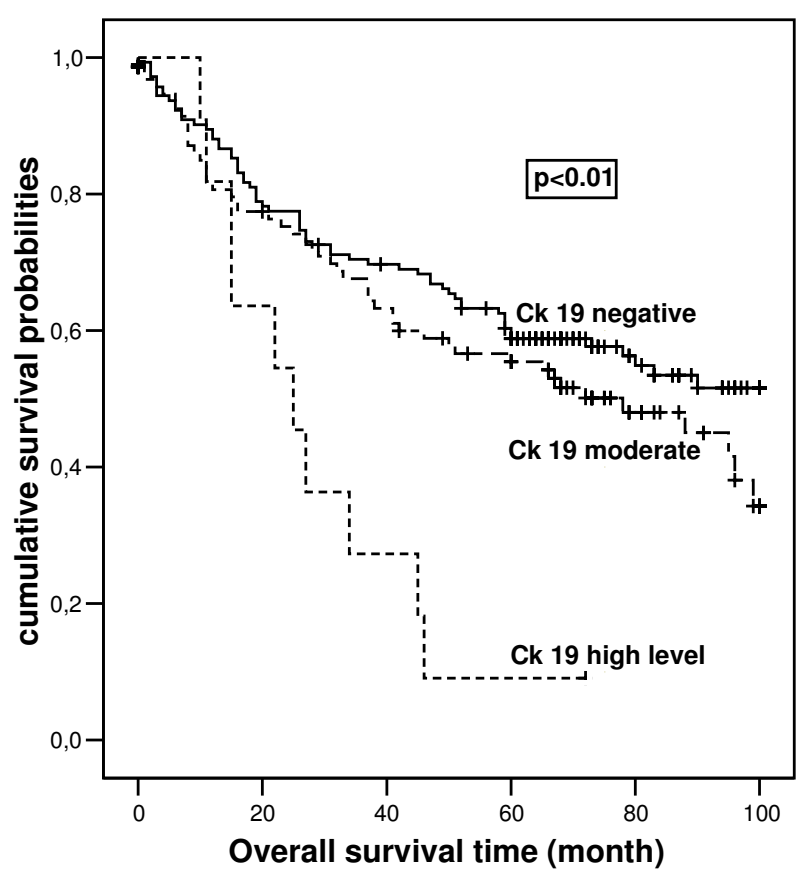

Figure 3

Overall survival of patient with oral squamous cell carcinoma in dependence on Cytokeratin 19 expression calculated by the Kaplan-Meier method.

parameters as nodal status, tumour size and $\mathrm{Ck} 8 / 18$ expression were indicated by Cox regression as independent predictors of survival prognosis.

\section{Discussion}

Intermediate filaments are central components of the intracellular skeleton. The expression of distinct intermediate filament proteins is tissue-specific and almost highly conserved during carcinogenesis. Intermediate filaments are interpreted as mere bystanders and markers of distinct intracellular, cell-specific regulation mechanisms. Several studies associated changes in intermediate filament expression with an altered cellular behaviour $[17,18]$.

The expression of high molecular weight cytokeratins, especially Ck 5 is a hallmark of squamous epithelium and is predominantly seen in the basal layers of stratified epithelium $[2,19]$. This cell layer is regarded as the anatomical localization of tissue specific stem/progenitor cells. Stem cells of stratified epithelium have been repeatedly described as the major cellular targets for cancer causing mutations and therefore might give in a long term rise to the development of SCC's. In the context of the existing literature it seems logical that SCC's retain and are pheno- 
Nodal negative OSCCs Cytokeratin 8/18 expression

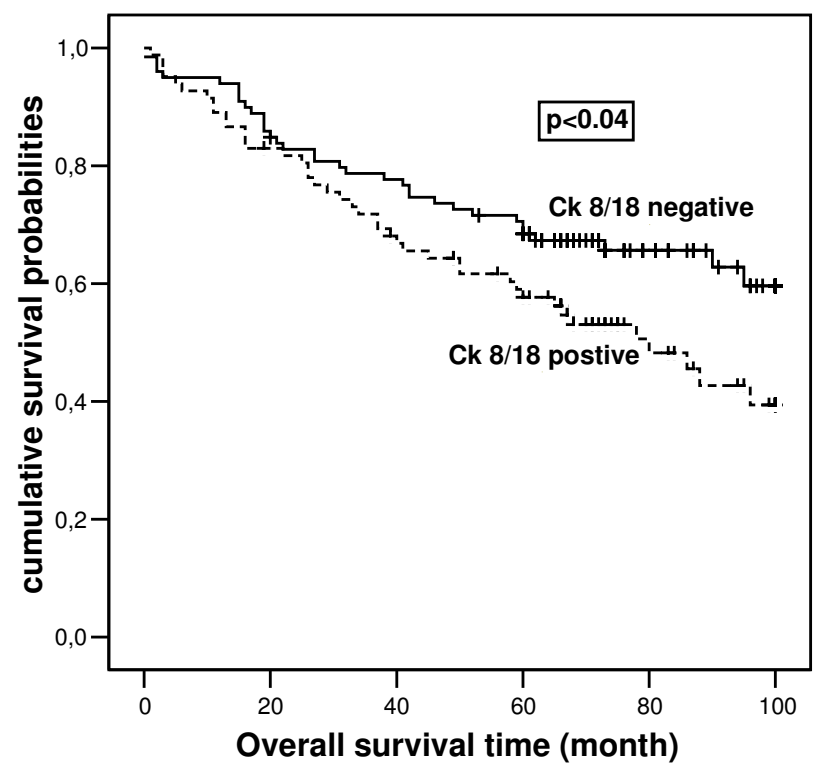

Figure 4

Overall survival of patient with nodal negative oral squamous cell carcinoma in dependence on Cytokeratin 8-18 expression calculated by the Kaplan-Meier method.

typically characterized by the expression of Ck 5. Adult healthy non-keratinizing stratified squamous epithelium of the upper aerodigestive tract express Ck 19 in basal layers but normally do not express Ck 8/18 [19]. Typical glandular cytokeratin $\mathrm{Ck} \mathrm{8/18}$ are normally not expressed in SCC's [2,4]. On the other hand, Ck 8 and 18 is expressed in fetal buccal mucosa and tongue epithelium till 27 weeks of gestation [20].

Our study shows in a series of more than 300, uniformly treated, primary SCC's of the oral cavity that the expres- sion of glandular cytokeratins such as Ck 8, 18 and 19, pinpoints a significant subgroup of SCC's. These carcinomas were associated with a poor prognosis, independently of the primary, preoperative lymph node status. It is important to stress that this held also true in multivariate analysis. This might be surprising on a first glance, since recent other clinico-pathological studies in SCC's of the oral cavity, especially with the focus on Ck 19 revealed inhomogeneous results [21-24]. This might on the one hand be due to significantly lower numbers of investigated tumour samples, and on the other hand caused by different techniques for the determination for the expression of cytokeratins 8/18 and 19 on the RNA and protein level. However, similar results to our findings have also been reported for skin carcinomas. Nevertheless, recent cell culture and studies with chemical carcinogens strongly support our results and revealed an important role of CK 8/18 in various steps of the pathogenesis and progression of SCC's. A spontaneous induction of Ck's 8 and 18 expression could be shown in SV40T-immortalized buccal mucosa cells, in tobacco induced leukoplakias and after introduction of v-H-Ras in epidermal mouse keratinocytes [25]. The transfection of buccal mucosa cells with a vector for Ck 8/18 resulted additionally in a significantly altered cellular morphology and increased cell motility - features associated with or being prerequisites for invasive tumour behaviour [10]. This was in line with previous experiments in melanoma cells [26]. The involvement of Ck 8/18 in SCC progression could further be demonstrated in chemically induced SCC's in the mouse skin. Whereas SCC's in mouse epidermis were Ck $8 / 18$ positive, non-invasive papillomas did not show this feature. However, the underlying disturbed intra- and extra cellular regulation mechanisms have been characterized not in detail yet. A central role for TGF-alpha via the Epidermal Growth Factor Receptor (EGFR) has been postulated $[27,28]$. In contrast, the induction of $\mathrm{Ck} 8 / 18$ expression in a similar experimental setting has also been described for TGF-alpha deficient mice, with putative involvement of H-Ras [29]. In consequence, multiple

Table 5: Multivariate analysis of the time of survival in relation to the Cytokeratins expression and other prognostic factors

\begin{tabular}{lccc}
\hline Prognosticator & P value $^{\mathrm{a}}$ & RR $^{\mathrm{b}}$ & Confidance intervalc $^{\mathrm{c}}$ \\
\hline Tumor size & $* 0.036$ & 1.239 & $1.0-1.5$ \\
Nodal status & $* 0.000$ & 1.866 & $1.4-2.4$ \\
CK5/6 & 0.350 & 1.167 & $0.8-1.6$ \\
CKI & 0.931 & 0.985 & $0.7-1.4$ \\
CKI0 & 0.852 & 0.968 & $0.7-1.4$ \\
CKI9 & 0.227 & 1.163 & $0.9-1.5$ \\
CKI4 & 0.470 & 0.872 & $0.6-1.3$ \\
CK8/18 & $* 0.024$ & 1.487 & $1.1-2.1$
\end{tabular}

a The partially nonparametric regression model of Cox (1972) was used to evaluate the predictive power of vatious combinations of prognosticators in a multivariate manner.

$\mathrm{b} R \mathrm{R}$, relative risk

c $95 \%$ confidence interval 
pathways in the regulation of $\mathrm{Ck} 8 / 18$ are conceivably existing.

In general, cytokeratin expression patterns are highly-conserved. The above mentioned data from the literature showed that any change in the intermediate filament expression pattern reflects or underlies changes in substantial cellular properties. Strong homologies could also be shown in other carcinomas. In primary breast cancer, this could be demonstrated with different approaches. Down-regulated expression of cytokeratin Ck 8/18 was associated with increased metastatic properties and poor prognosis in breast cancer [30]. The expression of Ck 5 in a small subset of invasive breast cancers was in contrast associated with a poor prognosis [31].

Interestingly, the expression of vimentin, alone or in combination with $\mathrm{Ck} \mathrm{8/18}$ and 19, was not associated with poor prognosis or higher tumour stages. A multitude of studies gave evidence that the expression of vimentin in carcinomas has to be interpreted as a sign of an epithelialmesenchymal transition, associated with high tumour aggressiveness $[9,32]$. This association remains questionable, since the expression of vimentin has already been described in non-invasive premalignant lesions of squamous epithelium and cultured keratinocytes.

Finally, from a clinical point of view, our results show that the determination of the Cytokeratin 8-18 and 19-expression in SCC of the oral cavity harbours a significant prognostic potential. Our results seem to define subgroups of high-risk patients who might benefit from more intensive therapy or new therapeutic strategies like Cytokeratin 19 antibodies [33]. For human ovarian adenocarcinoma cells it could be shown that cytokeratins 18 could sensitize these cells to cisplatin [34]. These results demonstrate that modulating the expression of an intermediate filament protein results in sensitization to chemotherapeutic drugs. The subgroup of ck 8-18 positive squamous cell carcinoma may have a benefit by chemotherapy with cisplatin. Further investigations are necessary to determine the clinical impact of these hypotheses.

\section{Conclusion}

In conclusion, our results in a series of 308 primary, previously untreated SCC's of the oral cavity showed an important prognostic impact for the expression of glandular, simple cytokeratins in SCC's. Further studies have to unravel the underlying regulatory changes in order to allow the definition of specific therapies of these high risk cancer patients.

\section{Competing interests}

The author(s) declare that they have no competing interests.

\section{Authors' contributions}

TF: Project planning, data analysis and writing of the manuscript

RW: Project planning and critical appraisal of the manuscript

JP: Data analysis and critical appraisal of the manuscript

BB: Project planning, critical appraisal of the manuscript

UJ: Critical appraisal of the manuscript

PM: Data analysis and critical appraisal of the manuscript

DW: Project planning and critical appraisal of the manuscript

HB: Project planning, data analysis and writing of the manuscript

\section{References}

I. Moll R, Franke WW, Schiller DL, Geiger B, Krepler R: The catalog of human cytokeratins: patterns of expression in normal epithelia, tumors and cultured cells. Cell 1982, 3 I (I): I I-24.

2. Presland RB, Jurevic RJ: Making sense of the epithelial barrier: what molecular biology and genetics tell us about the functions of oral mucosal and epidermal tissues. J Dent Educ 2002, 66(4):564-74.

3. van der Velden LA, Manni J], Ramaekers FC, Kuijpers W: Expression of intermediate filament proteins in benign lesions of the oral mucosa. Eur Arch Otorhinolaryngol 1999, 256( I 0):514-9.

4. Lindberg K, Rheinwald JG: Suprabasal 40 kd keratin (K/9) expression as an immunohistologic marker of premalignancy in oral epithelium. Am J Pathol I989, I34(I):89-98.

5. Vernon AE, LaBonne C: Tumor metastasis: a new twist on epithelial-mesenchymal transitions. Curr Biol I4(I7):R7I9-2I. 2004 Sep 7

6. Santini D, Ceccarelli C, Taffurelli M, Pileri S, Marrano D: Differentiation pathways in primary invasive breast carcinoma as suggested by intermediate filament and biopathological marker expression. J Pathol 1996, I 79(4):386-9I.

7. Domagala W, Lasota J, Bartkowiak J, Weber K, Osborn M: Vimentin is preferentially expressed in human breast carcinomas with low estrogen receptor and high $\mathrm{Ki}-67$ growth fraction. $\mathrm{Am} J$ Pathol 1990, I36(I):219-27.

8. Ramaekers FC, Haag D, Kant A, Moesker O, Jap PH, Vooijs GP: Coexpression of keratin- and vimentin-type intermediate filaments in human metastatic carcinoma cells. Proc Natl Acad Sci U S A 1983, 80(9):2618-22.

9. de Araujo VC, Pinto Junior DS, de Sousa SO, Nunes FD, de Araujo NS: Vimentin in oral squamous cell carcinoma. Eur Arch Otorhinolaryngol 1993, 250(2): 105-9.

10. Raul U, Sawant S, Dange P, Kalraiya R, Ingle A, Vaidya M: Implications of cytokeratin $8 / 18$ filament formation in stratified epithelial cells: induction of transformed phenotype. Int J Cancer I | I(5):662-8. 2004 Sep 20

II. Robbins KT, Clayman G, Levine PA, Medina J, Sessions R, Shaha A, Som P, Wolf GT, American Head and Neck Society; American Academy of Otolaryngology - Head and Neck Surgery: Neck dissection classification update: revisions proposed by the American Head and Neck Society and the American Academy of Otolaryngology-Head and Neck Surgery. Arch Otolaryngol Head Neck Surg 2002, I 28(7):75 I-8.

12. Wittekind C, Greene FL, Hutter RVP, Klimpfinger M, Sobin LH: TNM Atlas. 5th edition. Springer Verlag Heidelberg; 2005.

13. Kononen J, Bubendorf L, Kallioniemi A, Barlund M, Schraml P, Leighton S, Torhorst J, Mihatsch MJ, Sauter G, Kallioniemi OP: Tissue 
microarrays for high-throughput molecular profiling of tumour specimens. Nat Med 1998, 4:844-847.

14. Packeisen J, Buerger H, Krech R, Boecker W: Tissue microarrays: a new approach for quality control in immunohistochemistry. J Clin Pathol 2002, 55(8):613-5.

15. Kaplan EL, Meier P: Nonparametric estimation from incomplete observations. J Am Stat Ass 1958, 53:457-48I.

16. Cox DR: Regression models and life-tables. JR Stat Soc $[B] 1972$, 34: $187-202$.

17. Watanabe S, Ichikawa E, Takahashi H, Otsuka F: Changes of cytokeratin and involucrin expression in squamous cell carcinomas of the skin during progression to malignancy. $\mathrm{Br} J$ Dermatol 1995, I 32(5):730-9.

18. Morgan PR, Su L: Intermediate filaments in oral neoplasia. I. Oral cancer and epithelial dysplasia. Eur J Cancer B Oral Oncol 1994, 30B(3): 160-6. Review

19. Chu PG, Weiss LM: Keratin expression in human tissues and neoplasms. Histopathology 2002, 40(5):403-39.

20. Vaidya MM, Sawant SS, Borges AM, Naresh NK, Purandare MC, Bhisey AN: Cytokeratin expression in human fetal tongue and buccal mucosa. J Biosci 2000, 25(3):235-42.

21. Crowe DL, Milo GE, Shuler CF: Keratin 19 downregulation by oral squamous cell carcinoma lines increases invasive potential. J Dent Res 1999, 78(6): 1256-63.

22. Depondt J, Shabana AH, Sawaf H, Gehanno P, Forest N: Cytokeratin alterations as diagnostic and prognostic markers of oral and pharyngeal carcinomas. A prospective study. Eur J Oral Sci 1999, I07(6):442-54.

23. Vora HH, Shah NG, Patel DD, Trivedi TI, Chikhlikar PR: Prognostic value of clinicopathological parameters in head and neck squamous cell carcinoma: a prospective analysis. $\mathrm{Br} J$ Cancer 1996, 73(4):53I-8.

24. Janot F, Klijanienko J, Russo A, Mamet JP, de Braud F, El-Naggar AK, Pignon JP, Luboinski B, Cvitkovic E: Prognostic value of clinicopathological parameters in head and neck squamous cell carcinoma: a prospective analysis. BrJ Cancer 1996, 73(4):531-8.

25. Hansson A, Bloor BK, Sarang Z, Haig Y, Morgan PR, Stark HJ, Fusenig $\mathrm{NE}$, Ekstrand J, Grafstrom RC: Analysis of proliferation, apoptosis and keratin expression in cultured normal and immortalized human buccal keratinocytes. Eur J Oral Sci 2003, I I I (I):34-4I.

26. Chu YW, Seftor EA, Romer LH, Hendrix MJ: Experimental coexpression of vimentin and keratin intermediate filaments in human melanoma cells augments motility. Am J Pathol 1996, I 48(I):63-9.

27. Inada $\mathrm{H}$, Izawa I, Nishizawa M, Fujita E, Kiyono T, Takahashi T, Momo $T$, Inagaki M: Keratin attenuates tumor necrosis factorinduced cytotoxicity through association with TRADD. J Cell Biol I 55(3):4I 5-26. Epub 200I Oct 29

28. Gilbert S, Loranger A, Daigle N, Marceau N: Simple epithelium keratins 8 and 18 provide resistance to Fas-mediated apoptosis. The protection occurs through a receptor-targeting modulation. J Cell Biol I54(4):763-73. 200I Aug 20

29. Oshima RG, Baribault $H$, Caulin C: Oncogenic regulation and function of keratins 8 and 18. Cancer Metastasis Rev 1996, | 5(4):445-7|

30. Woelfle U, Sauter G, Santjer S, Brakenhoff R, Pantel K: Down-regulated expression of cytokeratin 18 promotes progression of human breast cancer. Clin Cancer Res I 0(8):2670-4. 2004 Apr 15

31. Abd El-Rehim DM, Pinder SE, Paish CE, Bell J, Blamey RW, Robertson JF, Nicholson RI, Ellis IO: Expression of luminal and basal cytokeratins in human breast carcinoma. J Pathol 2004, 203(2):66|-7I.

32. Schaafsma HE, Van Der Velden LA, Manni J], Peters H, Link M, Rutter DJ, Ramaekers FC: Increased expression of cytokeratins 8, I8 and vimentin in the invasion front of mucosal squamous cell carcinoma. I Pathol 1993, I70(I):77-86.

33. Yuan CC, Huang HC, Tsai LC, Ng HT, Huang TS: Cytokeratin- 19 associated with apoptosis and chemosensitivity in human cervical cancer cells. Apoptosis 1997, 2(I): I0I-5.

34. Parekh HK, Simpkins H: The differential expression of cytokeratin 18 in cisplatin-sensitive and -resistant human ovarian adenocarcinoma cells and its association with drug sensitivity. Cancer Res 55(22):5203-6. 1995 Nov 15

\section{Pre-publication history}

The pre-publication history for this paper can be accessed here:

http://www.biomedcentral.com/1471-2407/6/10/prepub
Publish with Biomed Central and every scientist can read your work free of charge

"BioMed Central will be the most significant development for disseminating the results of biomedical research in our lifetime. "

Sir Paul Nurse, Cancer Research UK

Your research papers will be:

- available free of charge to the entire biomedical community

- peer reviewed and published immediately upon acceptance

- cited in PubMed and archived on PubMed Central

- yours - you keep the copyright
BioMedcentral 